\title{
Oscillating mitotic newt lung cell kinetochores are, on average, under tension
} and rarely push

\author{
Jennifer C. Waters ${ }^{1}$, Robert V. Skibbens ${ }^{2}$ and E. D. Salmon ${ }^{1, *}$ \\ ${ }^{1}$ University of North Carolina, Department of Biology, 607 Fordham Bldg, CB\# 3280, Chapel Hill, NC 27599-3280, USA \\ 2Johns Hopkins University School of Medicine, Department of Molecular Biology and Genetics, 617 Hunterian Bldg, 725 N. Wolfe \\ St, Baltimore, MD 21205, USA
}

*Author for correspondence

\section{SUMMARY}

Experimentally introduced tension on kinetochores and their centromeres has been shown to stabilize kinetochore attachment to microtubules, modify kinetochore directional instability, and regulate cell-cycle progression into anaphase. In mitosis, kinetochore tension and the stretch of centromere chromatin are produced by the movement of sister kinetochores toward opposite poles and astral ejection forces on the chromosome arms. However, newt lung cell kinetochores oscillate between poleward and away from the pole motility states throughout mitosis, indicating kinetochores are not under constant tension. To test whether kinetochores are under net tension while they are oscillating, and how often they are under compression and pushing into the chromosome, we measured the distance between sister kinetochores in newt lung cells using both video-enhanced differential interference contrast microscopy (VE-DIC) and immunofluorescence microscopy. We found that for chromosomes in which sister kinetochores are attached to opposite spindle poles, centromeres are, on average, stretched $(2.2 \mu \mathrm{m}$ in living cells and $1.8 \mu \mathrm{m}$ in fixed cells) with respect to the inter-kinetochore 'rest' length $(1.1 \mu \mathrm{m}$ in living and fixed cells). For chromosomes in which only one kinetochore is attached to the spindle, the centromere chromatin associated with the tethered kinetochore is, on average, stretched to approximately half of the average inter-kinetochore distance measured for chromosomes in which both kinetochores are attached. We conclude that while newt lung cell kinetochores oscillate between states of $P$ and AP movement, they are under tension approximately $90 \%$ of the time and under compression less than $6 \%$ of the time.

Key words: Kinetochore directional instability, Tension, Mitosis, $3 \mathrm{~F} 3 / 2$

\section{INTRODUCTION}

To ensure accurate chromosome segregation, sister chromatids must attach to opposite poles of the bipolar spindle. Initially, a kinetochore on a chromosome captures microtubules from one spindle pole (i.e. mono-orientation). When the opposing sister kinetochore captures microtubules from the other spindle pole (i.e. bi-orientation), the chromosome congresses to the metaphase plate. Various forces act on the chromosome as it associates with the spindle; both kinetochore poleward (P) motion (Skibbens et al., 1993) and poleward flux of the microtubule lattice (Mitchison and Salmon, 1992) act to pull chromatids poleward, while kinetochore movement away from the pole (AP motion; Skibbens et al., 1993) and astral microtubule ejection forces on the chromosome arms (reviewed by Rieder and Salmon, 1995; Fuller, 1995; Vernos and Karsenti, 1996) push the chromatids toward the metaphase plate. It has been proposed that the result of these forces is a net tension across the centromere chromatin between sister kinetochores on bi-oriented chromosomes.

There is evidence that tension across centromeres could have roles in several aspects of cell division. Nicklas and Koch (1969) used micromanipulation to show that tension stabilizes kinetochore attachment to microtubules in meiotic grasshopper spermatocytes. In both mitotic and meiotic systems, the direction of kinetochore motility is sensitive to tension (Nicklas, 1977; Hays, 1985; Skibbens et al., 1995). In fact, laser ablation of one metaphase sister kinetochore or the centromere region between sisters results in anaphase A-like chromosome movement (McNeill and Berns, 1981; Rieder et al., 1986; Hays and Salmon, 1990; Ault et al., 1991; Skibbens et al., 1995). In addition, bi-oriented newt chromosomes require a stiff centromere linkage to produce coordinated sister kinetochore motility; weakening of the intervening centromeric chromatin via laser ablation results in uncoordinated sister kinetochore motility and movement away from the metaphase plate (Skibbens et al., 1995). Kinetochore tension has also been proposed to comprise part of a spindle assembly checkpoint that monitors spindle formation and chromosome alignment, and regulates when a cell exits mitosis and enters interphase (reviewed by McIntosh, 1991; Gorbsky, 1995; Wells, 1996). Cells remain in metaphase until all chromosomes are properly attached to opposite spindle poles by kinetochore microtubules (Bajer and Mole-Bajer, 1956; Zirkle, 1970; Rieder and Hard, 1990; Nicklas and Arana, 1992; Rieder and Palazzo, 1992; 
Wells and Murray, 1996). In meiotic grasshopper spermatocytes, exogenous tension exerted along a mono-oriented chromosome results in a precocious exit from meiosis correlated with a decrease in kinetochore phosphorylation (Gorbsky and Ricketts, 1993; Nicklas et al., 1995).

However, several reports for both mitotic and meiotic systems indicate that kinetochores attached to polar microtubules oscillate between AP and P motility states (Seto et al., 1969; Mole-Bajer et al., 1975; Roos, 1976; Bajer, 1982; PickettHeaps et al., 1982; Rieder et al., 1986; Wise et al., 1991; Skibbens et al., 1993; Cassimeris et al., 1994). It is possible that centromeres and their kinetochores are not under constant tension but oscillate between states of compression and tension during kinetochore $\mathrm{AP}$ and $\mathrm{P}$ movements, respectively. AP movement has been shown to be capable of pushing into and compressing the centromeric chromatin (Bajer, 1982; Skibbens et al., 1993; Cassimeris et al., 1994). Therefore, it was unclear what fraction of the time the kinetochores of oscillating chromosomes are under net tension and what fraction of the time they are under compression and pushing on the chromosome.

Nicklas has shown that grasshopper spermatocyte "chromosomes are elastic; they spring back after they are stretched by the spindle or by a micromanipulation needle' (reviewed by Nicklas, 1988). In their studies of the initial stages of chromosome attachment to the mitotic spindle, Alexander and Rieder (1991) showed that microtubules attach to and 'tug' on kinetochores, stretching the kinetochore region poleward. These initial attachments are sometimes broken, and as a result the kinetochore region recoils. This demonstrates that newt centromeric chromatin is elastic. Therefore, we reasoned that if an average centromere stretch is maintained during normal kinetochore oscillations (Skibbens et al., 1993), the kinetochores and centromeres must be under tension. To determine if centromeres are stretched during kinetochore oscillations, we measured inter-kinetochore distances of chromosomes in living and fixed mitotic newt lung cells and compared those values to inter-kinetochore lengths of unattached chromosomes. We conclude that kinetochores tethered to microtubules are, on average, under tension and are only rarely compressed.

\section{MATERIALS AND METHODS}

\section{Tissue culture}

Taricha granulosa (newt) lung cells were cultured in Rose chambers as described by Rieder and Hard (1990). Cells were maintained with half-strength L-15 medium supplemented with fetal bovine serum and antibiotics, at $24^{\circ} \mathrm{C}$.

\section{High resolution VE-DIC microscopy}

Prior to observation, Rose chambers were disassembled and the coverslip inverted onto a glass slide containing double-stick tape spacers. Fresh medium was introduced into the chamber and the coverslip was then sealed onto the slide with VALAP (1:1:1 of vaseline, lanolin, and paraffin). Differential interference contrast (DIC) images were obtained and time-lapse recorded as described by Skibbens et al. (1993). High resolution images were obtained using an optical bench microscope (Walker et al., 1990) equipped with a Zeiss $\times 100 / 1.25$ NA Plan objective, a Zeiss 1.4 NA condenser, and Zeiss DIC Wollaston prisms. The microscope was further equipped with Zeiss heat-reflecting, Zeiss heat-cut, and Omega (Omega Optics, Burlington, VT) green interference filters ( $540 \mathrm{~nm}$ center wavelength, $20 \mathrm{~nm}$ bandwidth) placed before the condenser lens.

\section{Video equipment}

Video images were generated as described by Skibbens et al., 1993. A C2400 Hamamatsu Newvicon video camera was used to obtain images of chromosome movements in living cells. Images were recorded by time lapsing the camera signal (1 frame every 2 or 4 seconds) into a Panasonic TQ2028F Optical Memory Disc Recorder (OMDR) for analysis. To protect the cell from photodamage, a shuttering mechanism blocked the illumination beam between exposures.

\section{Tracking and analyses of DIC images}

Tracking and analyses were performed as described by Skibbens et al. (1993). Computer-generated $8 \times 8$ pixel cursors were superimposed on the images. Gray values for each pixel in an $8 \times 8$ array were used as a template to search consecutive images on the OMDR. The computer recorded frame number, $x, y$ coordinates for each object, and time. These data were later converted to distance over time plots and analyzed using the in-house program, Single Frame Movement (SFM).

\section{Immunofluorescence}

Cells were fixed and processed for indirect immunofluorescence microscopy as described by Gorbsky and Ricketts (1993). Cells were rinsed in PHEM (60 mM Pipes, $25 \mathrm{mM}$ Hepes at pH 7.0, $10 \mathrm{mM}$ EGTA, and $2 \mathrm{mM} \mathrm{MgCl}_{2}$ ) and then simultaneously extracted and fixed in $0.5 \%$ Triton X-100, 0.5\% formaldehyde, and $100 \mathrm{nM}$ microcystin in PHEM for 5 minutes. The cells were then fixed further in $1.0 \%$ formaldehyde in PHEM for 15 minutes. After rinsing for 20 minutes in MBST (10 $\mathrm{mM}$ Mops at $\mathrm{pH} 7.4,150 \mathrm{mM} \mathrm{NaCl}$ with $0.05 \%$ Tween-20), cells were blocked with $20 \%$ boiled goat serum in MBS (10 mM Mops at $\mathrm{pH} 7.4$, $150 \mathrm{mM} \mathrm{NaCl}$ ) for 30 minutes at $37^{\circ} \mathrm{C}$ and labeled for 45 minutes at room temperature with an ascites preparation of $3 \mathrm{~F} 3 / 2$ monoclonal antibody (Cyert et al., 1988; a gift from Dr G. J. Gorbsky, University of Virginia) diluted 1:1,000 in 5\% boiled goat serum in MBS. After rinsing in MBST for 20 minutes, the cells were incubated for 30 minutes at room temperature with a 1:200 dilution of TRITC-conjugated goat anti-mouse $\mathrm{IgG}$ in $5 \%$ boiled goat serum, rinsed for 20 minutes in MBST, and mounted in an anti-bleaching medium (50\% PBS, 50\% glycerol containing $n$-propyl gallate). For measurements of the resting state of the kinetochore, cells were treated with $15 \mu \mathrm{g} / \mathrm{ml}$ nocodazole in medium for 45 minutes prior to fixation. For control experiments, cells were treated with Lambda Phosphatase (New England BioLabs, Beverly MA) for 30 minutes at $37^{\circ} \mathrm{C}$ after fixation.

\section{Fluorescence microscopy}

Cells processed for immunofluorescence were viewed with a multimode digital fluorescence microscope system (Salmon et al., 1994). A Nikon Microphot FX-A microscope equipped with a $60 \times / 1.4$ NA Phase 4 objective was used. Images were captured with a Hamamatsu C4880 cooled CCD digital camera and routed to Metamorph image processing software (Universal Imaging Corp., Media, PA). The Metamorph software and a Ludl stepping motor were used to obtain $\mathrm{Z}$-series optical sections of cells in $0.5 \mu \mathrm{m}$ steps. Images were stored as stacks on optical disk cartridges with a Pinnacle Micro PMO-650 drive. Digital images were labeled using ClarisDraw (Claris Corp., Santa Clara, CA) and printed with a Tektronix Phaser II SDX dyesublimation printer (Wilsonville, OR).

\section{Distance measurements of immunofluorescence samples}

Metamorph tracking software was calibrated with a stage micrometer and used to measure the inter-kinetochore distance in $3 F 3 / 2$ labeled cells stored as stacks. When suitable kinetochore pairs were identified, the image was zoomed $200-400 \%$ to facilitate accurate placement of computer generated cursors over the center of each kinetochore. In cases where sister kinetochores were not in the same focal plane, the focal plane containing the brightest intensity kinetochore staining for each kinetochore in a sister pair was identified and the $z$-axis distance between focal planes recorded. The projected distance between sister 
kinetochores in the $y$-axis was measured directly. Results for the two distances were imported into Microsoft Excel and the total inter-kinetochore distance was calculated using the Pythagorean theorem (i.e. $\left.d^{2}=(y)^{2}+(z)^{2}\right)$.

\section{RESULTS}

\section{3f3/2 labels mitotic newt lung cell kinetochores, chromatin and centrosomes}

In mitotic PtK cells (Gorbsky and Ricketts, 1993) and meiotic grasshopper spermatocytes (Nicklas et al., 1995) the monoclonal antibody $3 \mathrm{~F} 3 / 2$ recognizes phosphoepitopes at the spindle poles and kinetochores. In these cell types 3F3/2 labels kinetochores differentially; the epitope at the kinetochore appears to be cell cycle regulated and tension-sensitive. We found that, in fixed newt lung cells, 3F3/2 labels centrosomes and kinetochores throughout mitosis with equal intensity regardless of kinetochore association with spindle microtubules or placement at the spindle equator (Fig. 1). At the same concentration used to label PtK and grasshopper cells $(1: 1,000), 3 \mathrm{~F} 3 / 2$ also stains the periphery of the chromatin (Fig. 1A-C). At higher dilutions (1:25,000-100,000), chromatin labeling is lost while kinetochores and centrosomes remain uniformly labeled (Fig. 1D-F). At dilutions higher than 1:100,000, kinetochore labeling cannot be seen (data not shown). As is the case with PtK and grasshopper cells, 3F3/2 staining in newts was lost when the cells were treated with phosphatase after fixation (data not shown). In addition, when phosphatase inhibitor was excluded from the fixation/extraction step, labeling intensity decreased (data not shown). Since $3 F 3 / 2$ labels kinetochores uniformly throughout mitosis in newt, it makes an excellent newt kinetochore/chromosome marker, and was therefore used in this study.

\section{Centromere rest length}

Before we could ascertain whether centromeres are stretched during kinetochore oscillations, we had to measure the distance between sister kinetochores at 'rest' (i.e. when kinetochores are not attached to microtubules). Newt lung cells were treated with medium containing $15 \mu \mathrm{g} / \mathrm{ml}$ nocodazole to induce microtubule depolymerization. Prophase cells were chosen and monitored until entry into mitosis, as evidenced by nuclear envelope breakdown (NEB). The cells were left in nocodazole for an additional 30-45 minutes after NEB to ensure that any maturation processes that occur at the kinetochore and centromere were completed.

The distance between sister kinetochores was measured in two ways. In the first method, sister kinetochore pairs on chromosomes were visualized using high resolution VE-DIC microscopy. The images were recorded onto an OMDR and distances were measured using a semi-automatic tracking program (Salmon et al., 1991; Skibbens et al., 1993). Only chromosomes that were situated planar to the optical section
Fig. 1. Mitotic newt lung cells labeled with the monoclonal antibody $3 \mathrm{~F} 3 / 2$. In this cell type, $3 \mathrm{~F} 3 / 2$ labels kinetochores uniformly throughout mitosis. Arrows point to centrosomes. The centrosome in E is slightly out of focus. (A) Prophase, (B) metaphase, and (C) anaphase newt lung cells labeled with $3 \mathrm{~F} 3 / 2$ diluted $1: 1,000$. At this antibody concentration, the periphery of the chromatin is labeled. (D) Prophase, (E) metaphase, and $(\mathrm{F})$ anaphase newt lung cells labeled with $3 \mathrm{~F} 3 / 2$ diluted 1:50,000. All images are the same magnification. Bar, $10 \mu \mathrm{m}$.
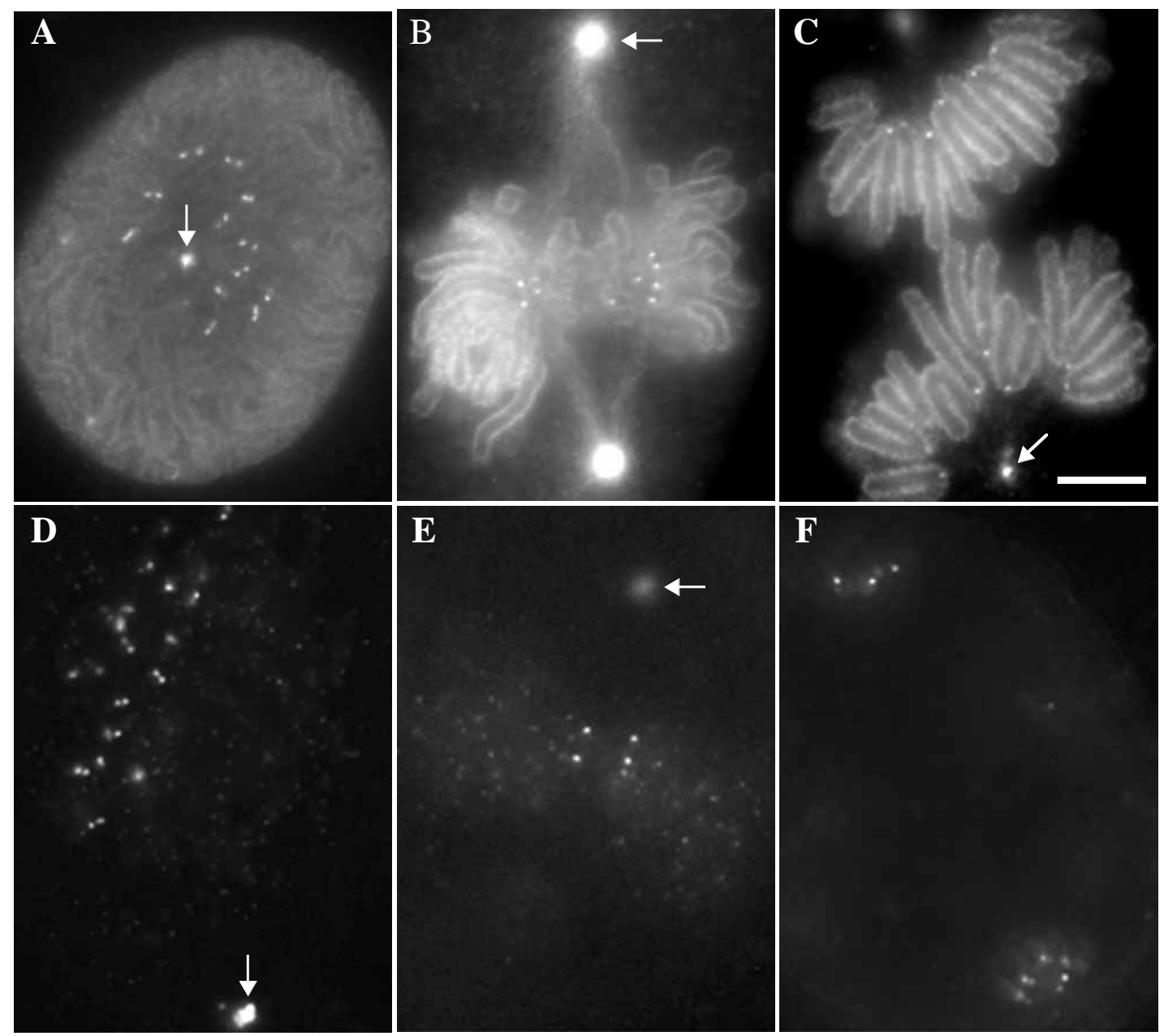

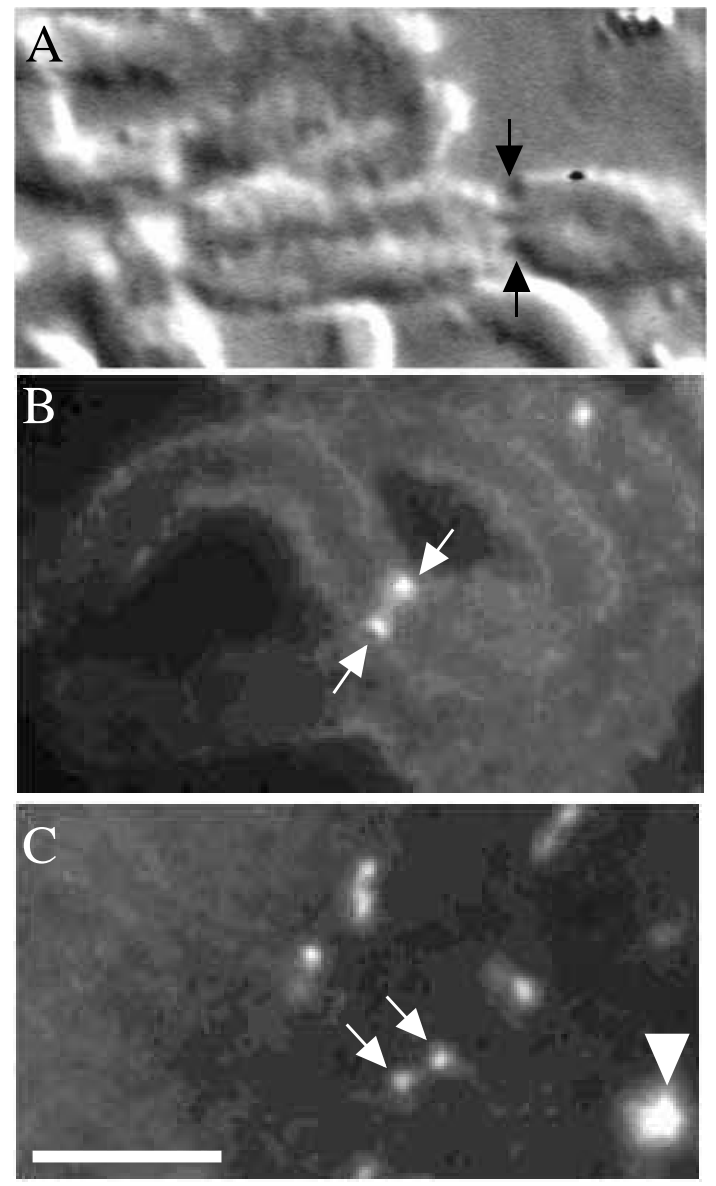

$\mathrm{D}$

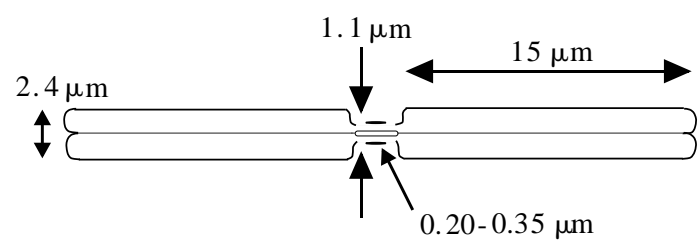

Fig. 2. Newt lung centromeres at 'rest'. (A) VE-DIC micrograph of a sister kinetochore pair in a nocodazole-treated newt lung cell. Newt lung cells were treated with $15 \mu \mathrm{g} / \mathrm{ml}$ nocodazole in prophase and allowed to proceed into mitosis. 45 minutes after NEB, the outer surfaces of the chromosome primary constriction (arrows) where each sister kinetochore pair resides is clearly visible.

(B) Immunofluorescence micrograph of a sister kinetochore pair in a nocodazole treated newt lung cell. Newt lung cells were treated with 15 $\mu \mathrm{g} / \mathrm{ml}$ nocodazole for 45 minutes and processed for immunodetection using the $3 \mathrm{~F} 3 / 2$ antibody. A sister kinetochore pair (arrow at each kinetochore) is strongly stained while the chromosome surface is lightly stained. Single dots (no arrows) are kinetochores whose sisters are in a different focal plane. (C) Immunofluorescence micrograph of a sister kinetochore pair in a prophase newt lung cell. A sister kinetochore pair (arrow at each kinetochore) and a centrosome (arrowhead) are visible in this focal plane. (D) Schematic diagram summarizing data obtained from VE-DIC and immunofluorescence microscopy regarding average dimensions of a newt lung cell chromosome free of MTs. Interkinetochore distance is $1.1 \pm 0.17 \mu \mathrm{m}$ (49 samples); chromosome arm width is $2.4 \pm 0.23 \mu \mathrm{m}$ (5 samples), and centromere-to-telomere distance is about $15.2 \pm 2.2 \mu \mathrm{m}$ (7 samples). Kinetochore diameter $(0.20-0.35$ $\mu \mathrm{m})$ was determined by Rieder and Hard (1990) and Cassimeris et al. (1994). All images are at the same magnification. Bar, $5 \mu \mathrm{m}$. and provided clear images of each sister kinetochore at the chromosome primary constriction were measured (Fig. 2A). The average inter-kinetochore rest length in nocodazole was $1.1 \pm 0.11 \mu \mathrm{m}$, based on duplicate trial measurements performed on 4 chromosomes (Table 1).

In the second method, we measured the distance between sister kinetochores at rest using indirect immunofluorescence microscopy. Mitotic newt lung cells were treated with nocodazole as described above and processed for immunofluorescence using the antibody $3 \mathrm{~F} 3 / 2$ (Fig. 2B). $z$-axis optical serial sections $(0.5 \mu \mathrm{m}$ steps $)$ through labeled cells were collected as 12 bit images by a cooled CCD and transferred to Metamorph software. This method enabled us to measure the inter-kinetochore distance on sister kinetochore pairs that were not in the same focal plane (see Materials and Methods). Based on the measurements made from 26 sister kinetochore pairs in 2 nocodazole-treated cells, the average inter-kinetochore rest length was $1.1 \pm 0.13 \mu \mathrm{m}$ (Table 1$)$.

Concerned that nocodazole treatment alters kinetochore morphology (reviewed by Rieder and Hard, 1990) and may affect the accuracy of our measurements, we measured the distance between resting sister kinetochores on chromosomes in untreated cells using the immunofluorescence techniques described above (Fig. 2C). Prior to nuclear envelope breakdown, prophase chromosomes are unable to interact with microtubules. Based on the measurements made from 40 sister kinetochore pairs in 3 late prophase cells, the average interkinetochore rest length was $1.1 \pm 0.17 \mu \mathrm{m}$ (Table 1). Occasionally in a prometaphase or metaphase cell, a chromosome can be found which is displaced away from the spindle in the cytoplasm, and not associated with any microtubules (i.e. a 'lost' chromosome). We measured the distance between sister kinetochores on 5 fixed and immunolabeled lost chromosomes, and found the average was $1.1 \pm 0.05 \mu \mathrm{m}$ (Table 1). Both of these measurements suggest that the inter-kinetochore distance measured in nocodazole treated cells is unaffected by any change in the kinetochore ultrastructure that is induced by MT depolymerization.

\section{Centromeres on bi-oriented chromosomes are predominantly stretched during kinetochore oscillations}

If sister kinetochores stretch and compress their centromeres to similar extents during kinetochore oscillations, then the

Table 1. Summary of inter-kinetochore distance of measurements

\begin{tabular}{lcccc}
\hline Experiments & $n^{*}$ & $\begin{array}{c}\text { Average } \pm \text { s.d. } \\
(\mu \mathrm{m})\end{array}$ & Mode & Range \\
\hline Prophase & 40 & $1.1 \pm 0.07$ & 1.0 & $1.0-1.3$ \\
Lost Chromosome & 5 & $1.1 \pm 0.05$ & - & $1.0-1.1$ \\
Nocodazole (DIC) & 4 & $1.1 \pm 0.11$ & 1.1 & $0.9-1.3$ \\
Nocodazole (IMF) & 26 & $1.1 \pm 0.13$ & 1.1 & $0.9-1.3$ \\
& & & & \\
Bi-oriented (DIC) & $5 \dagger$ & $2.2 \pm 0.58$ & 1.5 & $0.6-5$ \\
Bi-oriented (IMF) & 63 & $1.8 \pm 0.35$ & 1.8 & $1.0-2.4$ \\
Mono-oriented (IMF) & 33 & $1.5 \pm 0.29$ & $1.3-1.4$ & $1.0-2.3$
\end{tabular}

$*_{n}=$ number of chromosomes for which sister kinetochore inter-kinetochore distances were measured.

$\uparrow 1,336$ measurements taken over a 44.5 minute time period for 5 chromosomes. 


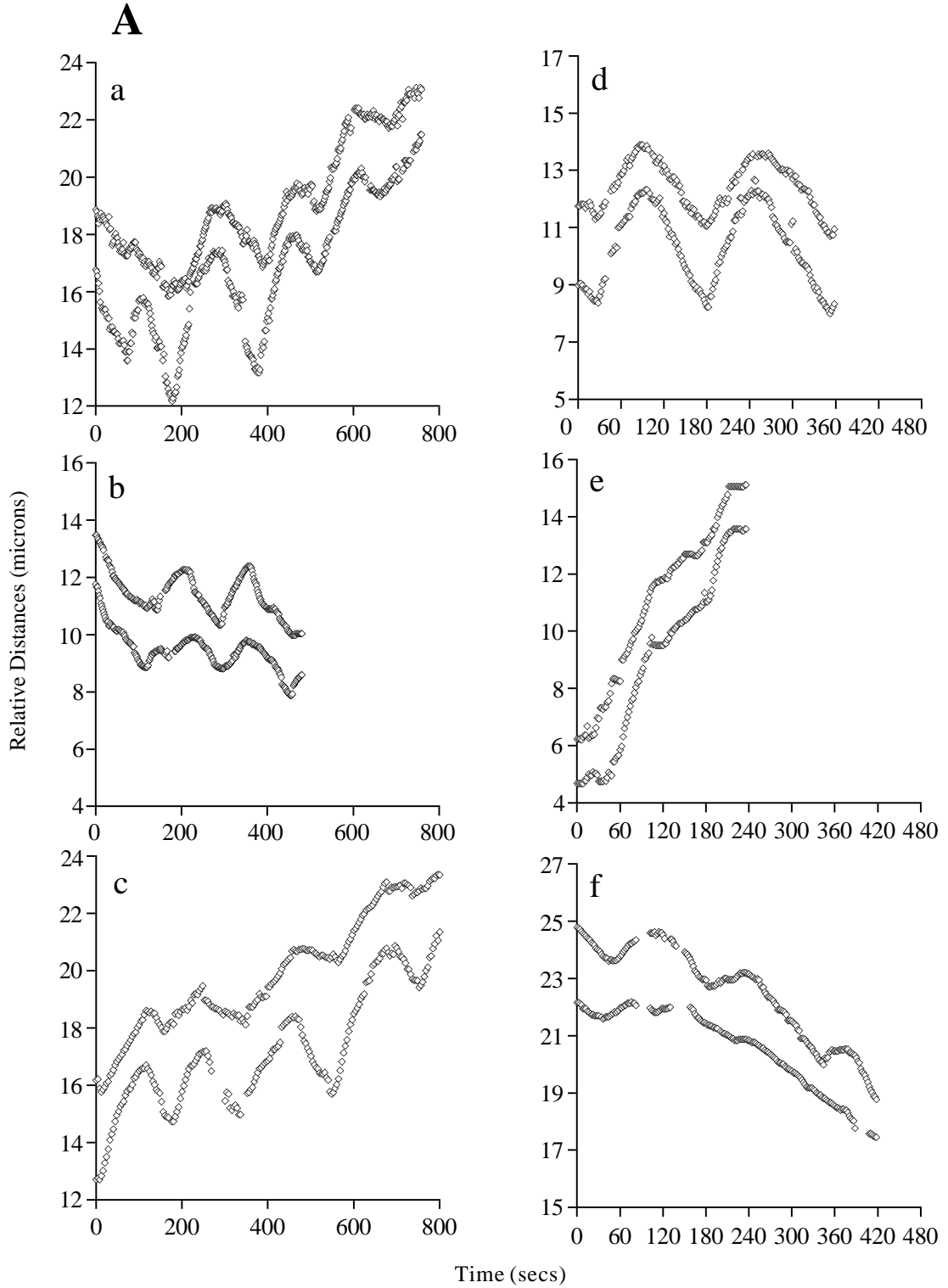

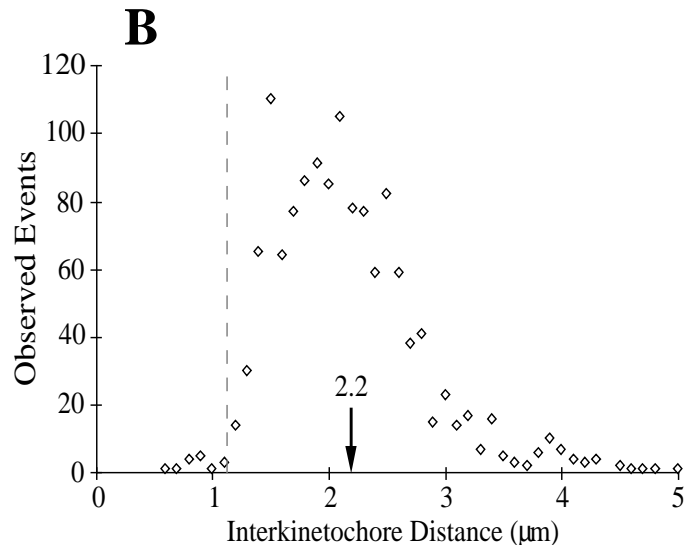

Fig. 3. Analyses of inter-kinetochore distances for bi-oriented chromosomes in newt lung cells using VE-DIC microscopy. (A) Distance-versus-time plots of several pairs of sister kinetochore movements in five cells, relative to one pole. Plots in $\mathrm{a}, \mathrm{b}, \mathrm{c}$, and $\mathrm{d}$ represent sister kinetochore pairs whose centromeres oscillated at a position near the spindle equator. Plots in e and f represent sister kinetochore pairs whose centromeres were congressing toward the spindle equator. Plots $\mathrm{c}$ and e were taken from Skibbens et al. (1993). For each time point, the distance between sister kinetochores was obtained and used to plot the histogram shown in B. (B) Histogram of inter-kinetochore distances measured from images obtained using VE-DIC microscopy plotted against frequency of occurrence. Distances were calculated for sister kinetochores in 5 cells with a 2 second sampling frequency over 2,672 seconds. Broken vertical line indicates inter-kinetochore rest length $(1.1 \mu \mathrm{m})$ for comparison. Arrow indicates the average value (2.2 $\mu \mathrm{m})$ for the data set. average inter-kinetochore length of bi-oriented chromosomes should be close to the inter-kinetochore rest length. Alternatively, if centromeres are predominately stretched or compressed during kinetochore oscillations, that bias should be reflected in the average inter-kinetochore distance. Sister kinetochore movements on bi-oriented chromosomes were tracked over time using VE-DIC microscopy and a semiautomated tracking system previously developed to track kinetochore motion at high resolution as described by Skibbens et al. (1993). For each chromosome analyzed, sister kinetochore movement, relative to one pole, was plotted and superimposed on one time axis (Fig. 3A). The distance between sister kinetochores for each 2 seconds time interval was then recorded and plotted against the number of occurrences. The resulting histogram (Fig. 3B) shows the distribution of inter-kinetochore distances for 5 pairs of sister kinetochores in 5 cells spanning over 44.5 minutes. The average inter-kinetochore distance was $2.2 \pm 0.58 \mu \mathrm{m}$, approximately double the distance measured for sister kinetochores at rest $(1.1 \mu \mathrm{m}$; Table 1$)$.
Indirect immunofluorescence was also used to measure the distance between sister kinetochores on bi-oriented chromosomes. Metaphase newt lung cells were fixed and processed for immunofluorescence using 3F3/2 (Fig. 4A). Distances between 63 pairs of labeled sister kinetochores in 6 cells were measured and plotted against the frequency of occurrence (Fig. 4B). Analysis of these data showed that the average inter-kinetochore distance in fixed cells is $1.8 \pm 0.35 \mu \mathrm{m}$, about $0.7 \mu \mathrm{m}$ over the inter-kinetochore rest length $(1.1 \mu \mathrm{m}$; Table 1$)$.

We found that centromeres on bi-oriented chromosomes were compressed (i.e. less than the rest length) only $\sim 1.6 \%$ of the time, at the rest length $6.4 \%$ of the time, and stretched (i.e. above the range of the rest length) $\sim 92 \%$ of the time (Fig. 4B). We were concerned that instead of compressing the centromere below the rest length, sister kinetochores actively pushing AP could rotate the centromere region. However, we found that only 2 out of the 5 sister kinetochore pairs that were at or below the rest length were tilted with respect to the pole to pole axis. 


\section{Kinetochores on mono-oriented chromosomes: one stretched, one relaxed}

Mono-oriented chromosomes typically have only one kinetochore attached to a spindle pole (Rieder et al., 1986), therefore tension cannot be generated by opposing poleward kinetochore forces. However, tension may be generated by polar ejection forces on the chromosome arms which oppose the poleward forces of the attached kinetochore (Cassimeris et al., 1994; reviewed by Rieder and Salmon, 1994). To determine if the attached kinetochore on a mono-oriented chromosome stretches its centromere, we processed newt lung cultures for immunofluorescence using $3 \mathrm{~F} 3 / 2$ and measured the distance between kinetochores on mono-oriented chromosomes (Fig. $5 \mathrm{~A})$. Values for 33 sister kinetochore pairs in 5 cells were plotted according to frequency of occurrence (Fig. 5B). Analysis of these data reveals that the average distance between sister kinetochores on mono-oriented chromosomes

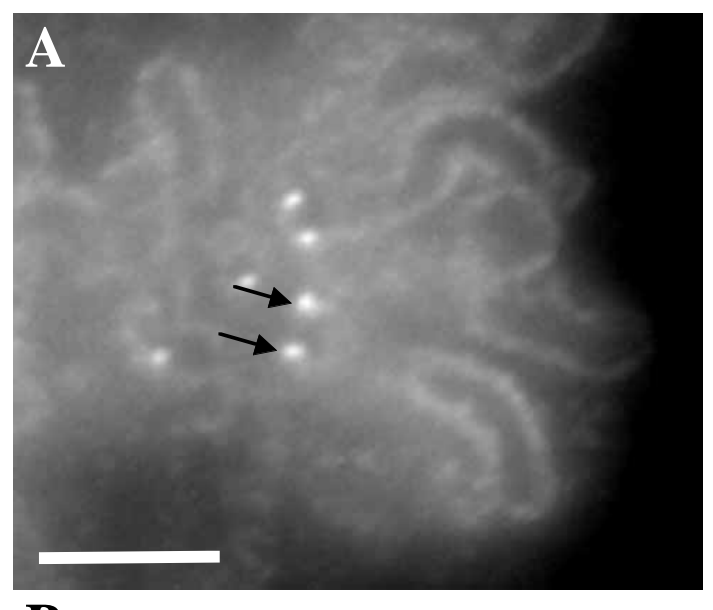

B

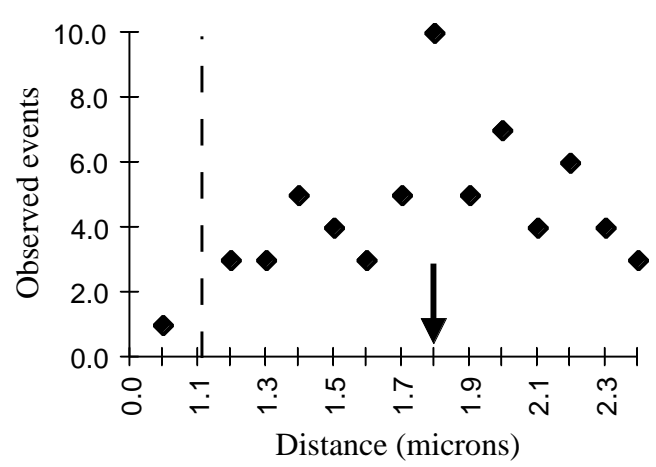

Fig. 4. Analysis of inter-kinetochore distances for bi-oriented chromosomes in newt lung cells using indirect immunofluorescence. (A) Immunofluorescence micrograph obtained using the $3 F 3 / 2$ antibody shows the brightly labeled kinetochores and the weakly labeled surface of the chromatin. Each kinetochore (arrows) in one sister kinetochore pair is labeled for clarity, although another pair is evident on the chromosome just above. Single dots (no arrows) are kinetochores whose sisters are in a different focal plane. Bar, $5 \mu \mathrm{m}$. (B) Histogram of inter-kinetochore distances for bi-oriented chromosomes obtained from image stacks of immunolabeled cells, plotted against frequency of occurrence. Broken vertical line indicates inter-kinetochore rest length $(1.1 \mu \mathrm{m})$ for comparison. Arrow indicates the average value $(1.8 \mu \mathrm{m})$ for the data set. was $1.5 \pm 0.29 \mu \mathrm{m}$. This distance is $0.4 \mu \mathrm{m}$ greater then the inter-kinetochore rest length $(1.1 \mu \mathrm{m}$; Table 1$)$ and statistically different from the distance between kinetochores on bioriented chromosomes (by Student's $t$-test, $P<1 \times 10^{-5}$ ). The centromeres on these mono-oriented chromosomes were compressed $\sim 3 \%$ of the time, at the rest length $10 \%$ of the time, and stretched $\sim 87 \%$ of the time (Fig. 5B).

\section{DISCUSSION}

\section{The newt kinetochore phosphoepitope recognized by $3 F 3 / 2$ is not cell cycle regulated}

The monoclonal antibody $3 \mathrm{~F} 3 / 2$ was originally generated against thio-phosphorylated mitotic Xenopus extracts (Cyert et

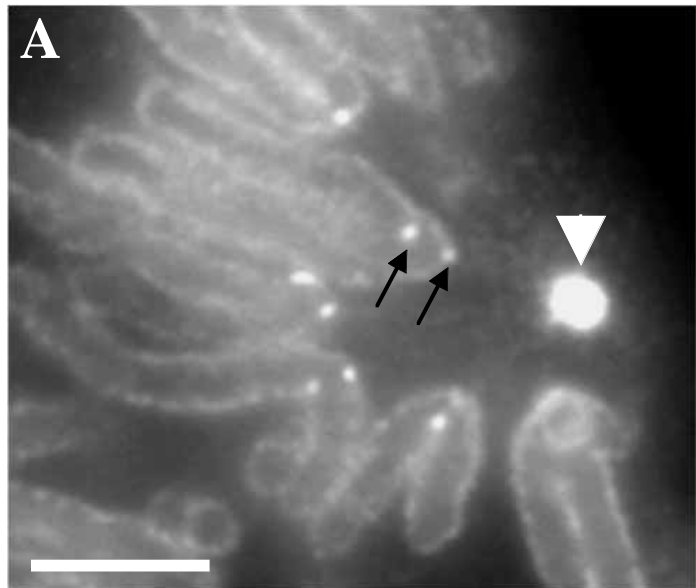

B

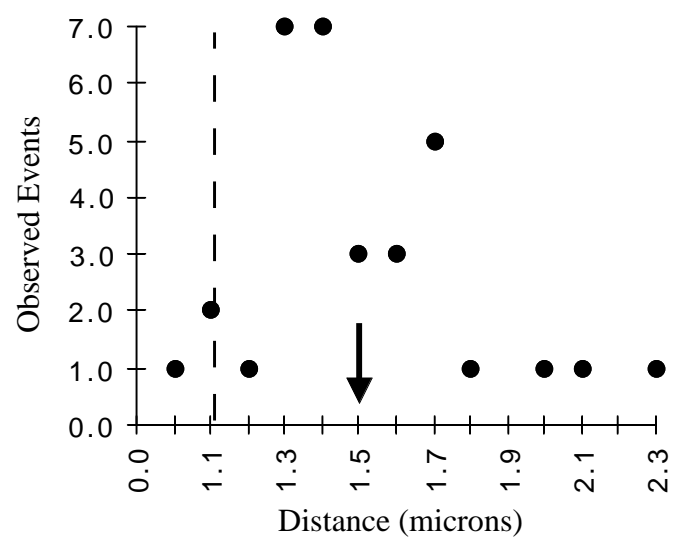

Fig. 5. Analyses of inter-kinetochore distances for mono-oriented chromosomes in newt lung cells using indirect immunofluorescence. (A) Immunofluorescence micrograph obtained using the $3 \mathrm{~F} 3 / 2$ antibody to visualize kinetochores shows a mono-polar half spindle with a strongly stained spindle pole (arrowhead) and weakly stained chromatin surface. Each kinetochore (arrows) of one sister kinetochore pair is labeled for clarity, although another pair is evident on the chromosome just below. Single dots (unlabeled) are kinetochores whose sisters are in a different focal plane. Bar, $5 \mu \mathrm{m}$. (B) Histogram of inter-kinetochore distances for mono-oriented chromosomes obtained from image stacks of immunolabeled cells plotted against frequency of occurrence. Dashed vertical line indicates inter-kinetochore rest length $(1.1 \mu \mathrm{m})$ for comparison. Arrow indicates the average value $(1.5 \mu \mathrm{m})$ for the data set. 
A Average Centromere Morphology

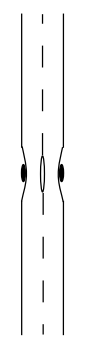

Rest

Mono-oriented

B

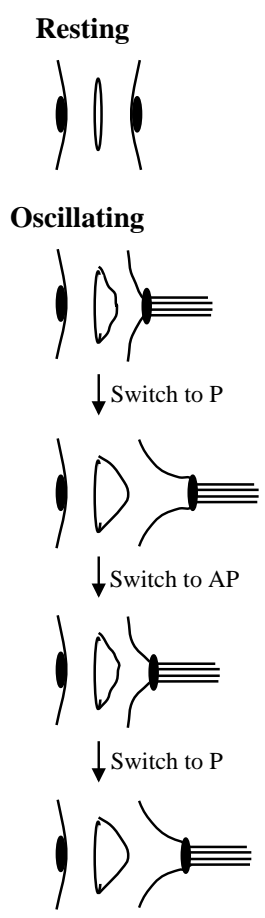

Fig. 6. Tense oscillations. (A) Sister chromatids are shown separated by a dotted line. At rest, the centromere is not under tension. When a chromosome becomes mono-oriented, the centromere associated with the unattached kinetochore remains at rest, while the attached kinetochore and its centromere become stretched. On a bi-oriented chromosome, both kinetochores are under tension $92 \%$ of the time. (B) Newt lung cell kinetochores remain under tension during the majority of oscillations. An oscillating mono-oriented chromosome is depicted in this cartoon. While resting, the kinetochore and centromere are not under tension. An attached kinetochore moves $\mathrm{P}$ until it reaches a threshold tension. It then moves AP, relieving the tension. The kinetochore switches back to P movement while it is still under a reduced amount of tension. Note: the size of the 'cleft' between sister chromatids is exaggerated for clarity (see Skibbens et al., 1993, Fig. 8, for DIC images of the cleft).

al., 1988). Previously, 3F3/2 has been shown to label the kinetochores of isolated $\mathrm{CHO}$ chromosomes after they had been treated with ATPYS (Wordeman et al., 1991; Hyman and Mitchison, 1991). 3F3/2 differentially labels kinetochores in mitotic PtK cells (Gorbsky and Ricketts, 1993) and meiotic grasshopper spermatocytes (Nicklas et al., 1995); kinetochores not yet attached to spindle microtubules label brightly while kinetochores on bi-oriented chromosomes label weakly or not at all (Gorbsky and Ricketts, 1993). Nicklas and coworkers (1995) showed that 3F3/2 labeling at the kinetochore decreases when tension is applied to the arms of mono-oriented chromosomes in meiotic invertebrate cells with a microneedle. Shortly after the application of tension the cells exit meiosis, thereby correlating cell cycle progression with tension-regulated dephosphorylation. Recently, Cambell and Gorbsky (1995) used electron microscopy to show that $3 F 3 / 2$ is localized between the inner and outer kinetochore plates in PtK cells.

In newt lung cells, however, we have found that 3F3/2 labels all kinetochores with similar intensity throughout mitosis. Concerned that a basal kinetochore staining masked our ability to detect changes in the kinetochore phosphorylation state, we performed serial dilutions of the antibody. Even at the highest possible dilution (without loss of kinetochore labeling), we did not see differential or changes in kinetochore labeling during mitosis (data not shown). Therefore, unlike PtK and grasshopper cells, either: (1) the kinetochore protein(s) that $3 F 3 / 2$ recognizes in newt lung cells remains phosphorylated throughout mitosis; or (2) any change in phosphorylation is masked by the presence of a more abundant kinetochore protein(s) which remains phosphorylated.

\section{Centromeres under tension}

Both immunofluorescence and VE-DIC microscopy methods were used to show that centromeres on bi-oriented chromosomes are, on average, stretched. In fixed cells (Fig. 4B), the average inter-kinetochore distance $(1.8 \mu \mathrm{m}$; Table 1$)$ is slightly diminished compared to the average inter-kinetochore distance (2.2 $\mu \mathrm{m}$; Table 1) measured in living cells (Fig. 3B). One explanation for this discrepancy is that when using immunofluorescence techniques, sister kinetochores at the metaphase plate which are far away from one another (i.e. greater than 2.5 $\mu \mathrm{m})$ are difficult to unmistakably identify as sisters. Since sisters occasionally reach very stretched states (Fig. 3B), this bias may lower the average inter-kinetochore distance for immunolabeled cells. Also, the cell lysis and fixation procedures used in immunolabeling may allow for some relaxation of the spindle structure and artificial diminution of the interkinetochore distance. Regardless, results obtained by both methods show that centromeres on bi-oriented chromosomes at the metaphase plate are predominantly stretched (Figs 3B, 4B), compared to inter-kinetochore rest lengths (Fig. 2).

For mono-oriented chromosomes, only one kinetochore is attached to microtubules and therefore capable of motility. In this case, centromere stretch is not due to differential sister kinetochore motility, but is produced by the attached kinetochore moving against polar microtubule ejection forces which act on the chromosome arms (see Rieder and Salmon, 1994, for review) and by forces generated by the poleward flux of their kinetochore microtubules (Mitchison and Salmon, 1992; Waters et al., 1996). Our data show that the average stretch across the centromeres of mono-oriented chromosomes (i.e. the inter-kinetochore distance minus the rest length) is approximately half the average stretch of bi-oriented chromosomes (by Student's $t$-test, $P=0.56$; Figs 4,5 ). The simplest conclusion from these observations is that attached kinetochores respond to tension across the centromeric DNA to which it is attached; a mono-oriented kinetochore moves $\mathrm{P}$ until its centromere is stretched to a threshold tension, then switches to the AP motility state (Fig. 6). Furthermore, the stretch attainable 
by a kinetochore before it switches to AP movement appears to be the same whether the antagonistic force is produced by sister kinetochore motility or by polar microtubule ejection forces and possibly poleward kinetochore microtubule flux (Rieder et al., 1986; Vernos and Karsenti, 1996).

These data are consistent with a spindle assembly checkpoint model where kinetochores at rest release a 'wait' signal which delays anaphase onset (reviewed by McIntosh, 1991; Gorbsky, 1995; Wells, 1996). In this model, kinetochores under tension stop sending the 'wait' signal. One monooriented PtK chromosome will delay the onset of anaphase until it achieves bi-orientation (Rieder et al., 1994) or until the unattached kinetochore is damaged with a laser microbeam (Rieder et al., 1995). As is predicted by this checkpoint model, our data reveal that, on average, sister kinetochores on bioriented chromosomes are stretched while on mono-oriented chromosomes only the centromere region of the attached kinetochore is stretched.

\section{Kinetochore AP movement relieves tension, but rarely pushes}

Skibbens and co-workers (1993) have shown that newt lung cell kinetochores exhibit directional instability; they abruptly switch between persistent AP and P constant velocity states. Analysis of centromere deformation during kinetochore motility showed that kinetochores are capable of pushing into the chromatin (i.e. compression). However, it was not clear how often kinetochores moving AP push into and compress the chromatin, and how often they move AP only enough to relieve the tension generated by previous $\mathrm{P}$ movement.

In this report, we show that the average inter-kinetochore length is stretched compared to the rest length (Figs 3B, 4B). The distance between the kinetochores is usually greater than the rest length; only rarely is the centromere compressed to less than the rest length $(1.6 \%$ of the time for bi-oriented chromosomes; $3 \%$ for mono-oriented chromosomes). Thus, the majority of AP movement occurs while the kinetochore is under tension, and therefore not actively pushing. However, since AP motility occurs at a constant velocity which is often independent of the velocity of the sister kinetochore (Fig. 3; Skibbens et al., 1993, 1995), it is likely that the kinetochore does not move passively AP but governs the rate at which moves. These data are consistent with the model proposed by Skibbens and co-workers (1995) in which kinetochores switch from AP to P constant velocity motility states when the centromere is under low tension (Fig. 6B).

\section{Note added in proof}

Our conclusions that kinetochores rarely push AP is supported by the recent studies of Khodjakov and Rieder (J. Cell Biol., 1996, in press) on kinetochore movements in P+K cells.

The authors thank Dr Gary Gorbsky for his generous gift of the 3F3/2 antibody as well as valuable advice. We thank Drs Andrew Murray, Bruce Nicklas, Kerry Bloom, Conly Rieder, and Lynn Cassimeris for insightful and stimulating discussions. We thank Paul Maddox for excellent technical assistance.

\section{REFERENCES}

Alexander, S. P. and Rieder, C. L. (1991). Chromosome motion during attachment to the vertebrate spindle: initial saltatory-like behavior of chromosomes and quantitative analysis of force production by nascent kinetochore fibers. J. Cell Biol. 113, 805-815.

Ault, J. G., Demarco, A. J., Salmon, E. D. and Rieder, C. L. (1991). Studies on the ejection properties of asters: astral microtubule turnover influences the oscillatory behavior and positioning of mono-oriented chromosomes. J. Cell Sci. 99, 701-710.

Bajer, A. and Mole-Bajer, J. (1956). Cine-micrographic studies on mitosis in endosperm II. Chromosome, cytoplasmic, and brownian movements. Chromosoma 7, 558-607.

Bajer, A. (1982). Functional autonomy of monopolar spindle and evidence for oscillatory movement in mitosis. J. Cell Biol. 93, 33-48.

Campbell, M. S. and Gorbsky, G. J. (1995). Microinjection of mitotic cells with the $3 \mathrm{~F} 3 / 2$ anti-phosphoepitope antibody delays the onset of anaphase. $J$. Cell Biol. 129, 1195-1204.

Cassimeris, L., Rieder, C. L. and Salmon, E. D. (1994). Microtubule assembly and kinetochore directional instability in vertebrate monopolar spindles: Implications for the mechanism of chromosome congression. $J$. Cell Sci. 107, 285-297.

Cyert, M. S., Scherson, T. and Kirschner, M. W. (1988). Monoclonal antibodies specific for thiophosphorylated proteins recognizes Xenopus MPF. Dev. Biol. 129, 209-216.

Fuller, M. T. (1995). Riding the polar winds: chromosomes motor down east. Cell 81, 5-8.

Gorbsky, G. J. and Ricketts, W. A. (1993). Differential expression of a phosphoepitope at the kinetochores of moving chromosomes. J. Cell Biol. 122, 1311-1321.

Gorbsky, G. J. (1995). Kinetochores, microtubules and the metaphase checkpoint. Trends Cell Biol. 5, 143-148.

Hays, T. S. (1985). The force-balance mechanism of chromosome congression. $\mathrm{PhD}$ thesis, University of North Carolina, Chapel Hill.

Hays, T. S and Salmon, E. D. (1990). Poleward force at the kinetochore in metaphase depends on the number of kinetochore microtubules. J. Cell Biol. 110, 391-404.

Hyman, A. A. and Mitchison., T. J. (1991). Regulation of the direction of chromosome movement. CSH Symp. Quant. Biol. 56, 745-750.

Li, X. and Nicklas, R. B. (1995). Mitotic forces control a cell-cycle checkpoint. Nature 373, 630-632.

McIntosh, J. R. (1991). Structural and mechanical control of mitotic progression. CSH Quant. Biol. 56, 613-619.

McNeill, P. A. and Berns, M. W. (1981). Chromosome behavior after laser microirradiation of a single kinetochore in mitotic PtK2 cells. J. Cell Biol. 88, 543-553.

Mitchison, T. J. and Salmon, E. D. (1992). Poleward kinetochore fiber movement occurs during both metaphase and anaphase-A in newt lung cell mitosis. J. Cell Biol. 119, 569-582.

Mole-Bajer, J., Bajer, A. and Owczarzak, A. (1975). Chromosome movements in prometaphase and aster transport in the newt. Cytobios 13, 4565.

Nicklas, R. B. and Koch, C. A. (1969). Chromosome micromanipulation III. Spindle fiber tension and the reorientation of mal-oriented chromosomes. $J$. Cell Biol. 43, 40-50.

Nicklas, R. B. (1977). Chromosome movement: facts and hypotheses. In Mitosis Facts and Questions (ed. M. Little et al.), pp. 150-15. SpringerVerlag, Berlin.

Nicklas, R. B. (1988) The forces that move chromosomes in mitosis. Annu. Rev. Biophys. Biophys. Chem. 17, 431-449.

Nicklas, R. B. and Arana, P. (1992). Evolution and the meaning of metaphase. J. Cell Biol. 102, 681-690.

Nicklas, R. B., Ward, S. C. and Gorbsky, G. J. (1995). Kinetochore chemistry is sensitive to tension and may link mitotic forces to a cell cycle checkpoint. J. Cell Biol. 130, 929-939.

Pickett-Heaps, J. D., Tippit, D. H. and Porter, K. R. (1982). Rethinking mitosis. Cell 29, 729-744.

Rieder, C. L., Davison, E. A., Jensen, L. C. W., Cassimeris, L. and Salmon, E. D. (1986). Oscillatory movements of mono-oriented chromosomes and their position relative to the spindle pole result from the ejection properties of the aster and half-spindle. J. Cell Biol. 103, 581-591.

Rieder, C. L. and Hard, R. (1990). Newt lung epithelial cells: Cultivation, use, and advantages for biomedical research. Int. Rev. Cytol. 122, 153-220.

Rieder, C. L. and Palazzo, R. E. (1992). Colcemid and the mitotic cycle. J. Cell Sci. 102, 387-392.

Rieder, C. L., Schultz, A., Cole, R. and Sluder, G. (1994). Anaphase onset in vertebrate somatic cells is controlled by a checkpoint that monitors sister kinetochore attachment to the spindle. J. Cell Biol. 127, 1301-1310. 
Rieder, C. L., Cole, R. W., Khodjakov, A. and Sluder, G. (1995). The checkpoint delaying anaphase in response to chromosome monoorientation is mediated by an inhibitory signal produced by unattached kinetochores. $J$. Cell Biol. 130, 941-948.

Rieder, C. L. and Salmon, E. D. (1994). Motile kinetochores and polar ejection forces dictate chromosome position on the vertebrate mitotic spindle. J. Cell Biol. 124, 223-233.

Roos, U. P. (1976). Light and electron microscopy of rat kangaroo cells in mitosis. Chromosoma 54, 363-385.

Salmon, E. D., Magers, S., Skibbens, R. and Gliksman, N. R. (1991). Videoenhanced differential interference contrast (VE-DIC) light microscopy: a method for semi-automatic object tracking. In Proc. 49th Annu. EMSA (ed. G. W. Bailey), pp. 238-239. San Francisco Press, San Francisco, CA.

Salmon, E. D., Inoue, T., Desai, A. and Murray, A. W. (1994). High resolution multimode digital imaging system for mitosis studies in vivo and in vitro. Biol. Bulletin 187, 231-232.

Seto, T., Kezer, J. and Pomerat, C. M. (1969). A cinematographic study of meiosis in salamander spermatocytes in vitro. Z. Zellforsch. 94, 407-424.

Skibbens, R. V., Skeen, V. P. and Salmon, E. D. (1993). Directional instability of kinetochore motility during chromosome congression and segregation in mitotic newt lung cells: a push-pull mechanism. J. Cell Biol. 122, 859-875

Skibbens, R. V., Rieder, C. L. and Salmon, E. D. (1995). Kinetochore motility after severing between sister centromeres using laser microsurgery: evidence that kinetochore directional instability and position is regulated by tension. J. Cell Sci. 108, 2537-2548.
Vernos, I. and Karsenti, E. (1996). Motors involved in spindle assembly and chromosome segregation. Curr. Opin. Cell. Biol. 8, 4-9.

Walker, R. A., Gliksman, N. R. and Salmon, E. D. (1990). Using videoenhanced differential interference contrast microscopy to analyze the assembly dynamics of individual microtubules in real time. In Optical Microscopy for Biology (ed. B. Herman and K. Jacobson), pp. 395-407. Wiley-Liss, Inc.

Waters, J. C., Mitchison, T. J., Rieder, C. L. and Salmon, E. D. (1996). The kinetochore microtubule minus-end disassembly associated with poleward flux produces a force that can do work. Mol. Biol. Cell 7, 1547-1558.

Wells, W. A. E. (1996). The spindle assembly checkpoint: aiming for a perfect mitosis, every time. Trends Cell Biol. 6, 228-234.

Wells, W. A. E. and Murray, A. W. (1996). Aberrantly segregating centromeres activate the spindle assembly checkpoint in budding yeast. $J$. Cell Biol. 133, 75-84.

Wise, D., Cassimeris, L., Rieder, C. L., Wadsworth, P. and Salmon, E. D. (1991). Chromosome fiber dynamics and congression oscillations in metaphase PtK2 cells at 23 degrees celsius. Cell Motil. Cytol. 18, 1-12.

Wordeman, L., Steuer, E. R., Sheetz, M. P. and Mitchison, T. (1991). Chemical subdomains within the kinetochore domain of isolated $\mathrm{CHO}$ mitotic chromosomes. J. Cell Biol. 114, 285-294.

Zirkle, R. E. (1970). Ultraviolet-microbeam irradiation of newt-cell cytoplasm: spindle destruction, false anaphase, and delay of true anaphase. Rad. Res. 41, 516-537.

(Received 18 June 1996 - Accepted 5 September 1996) 